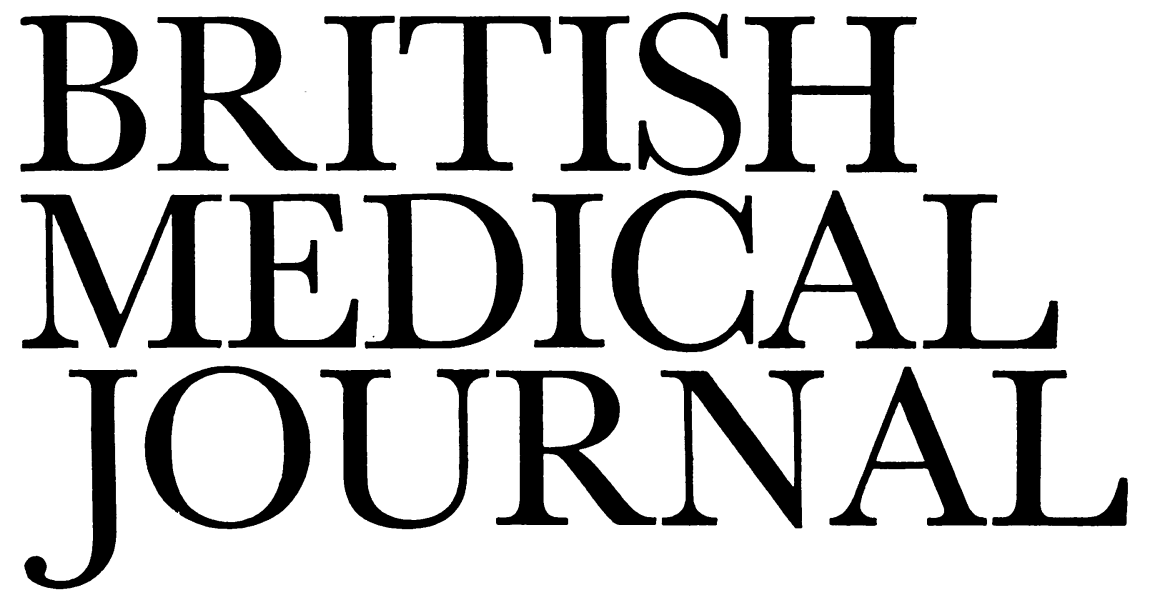

\title{
Opiate peptides, analgesia, and the neuroendocrine system
}

The isolation of the brain peptides with opiate-like analgesic actions-the enkephalins and the endorphins ${ }^{1}{ }^{2}$ - has led to study of their possible physiological actions. As yet it remains uncertain whether methionine-enkephalin and leucine-enkephalin function as neurotransmitters in the central and peripheral nervous systems or whether they merely modulate the release of transmitters at these synapses. ${ }^{3}$ The profound analgesia produced in animals by intraventricular methionineenkephalin and $\beta$-endorphin suggests that endogenous opiates may play a central part in modulating the perception of pain. ${ }^{4} 5$

The specific opiate antagonist naloxone has been used to test indirectly whether perception of pain can be altered in otherwise untreated individuals; for if enkephalins and endorphins do modulate pain and this system is continuously active naloxone should reduce the pain threshold. This certainly happens in rats. ${ }^{6}$ Studies on man have generally shown similar results, ${ }^{78}$ though possibly only people with high pain thresholds show naloxone-induced hyperalgesia. ${ }^{7}$ Naloxone increased postoperative pain in dental patients ${ }^{8}$; and it also increased pain in people who gained relief from a placebo treatment but not in non-responders to the placebo, suggesting a link between the endogenous opiates and the placebo response. ${ }^{9}$ Other studies, however, have shown conflicting results and interpretation remains difficult. Overall the data suggest that enkephalins and endorphins may be mobilised to modulate perception of pain in certain conditions, and such observations provide a physiological basis for the known variabilities in response to painful stimuli, which are usually regarded as due to "the attitude of the mind."

Beta-endorphin is found in the anterior pituitary as well as in the brain and in the same cells as adrenocorticotrophic hormone (ACTH) and $\beta$-lipotropin. All three are secreted in parallel during stress, ${ }^{10}$ possibly as an adaptive mechanism: the $\beta$-endorphin may help to relieve any pain the individual might incur. The methionine-enkephalin found in cerebrospinal fluid comes presumably from the central nervous system, but methionine-enkephalin in the plasma may be secreted specifically from the adrenal medulla into the circulation. ${ }^{11}$

Further evidence for the analgesic role of endogenous opiates has come from studies of the relief of pain from electrical stimulation of discrete areas of the diencephalon and brain stem. The analgesia has churacteristics similar to that produced by morphine: it can be partially antagonised by naloxone and both tolerance to and cross-tolerance with morphine may develop with indiscriminate overstimulation. In man electrical stimulation of the periventricular and periaqueductal grey matter has been used successfully for the treatment of chronic intractable pain. ${ }^{12-14}$ These brain areas are rich in opiate receptors, and microinjection of morphine into them is known to relieve pain. ${ }^{15}$ These data all suggest that electrical stimulation analgesia may be mediated through the release of endogenous opiates. There may be an increase of enkephalin-like material and $\beta$-endorphin in the ventricular cerebrospinal fluid after electrical stimulation of periaqueductal grey matter, ${ }^{1617}$ but substances have not yet been fully identified in the cerebrospinal fluid owing to lack of specificity in the assays used.

Acupuncture analgesia has been used for centuries in China, and recent Western studies have confirmed that it induces an increase in the pain threshold in man. How acupuncture works remains uncertain, but the finding that naloxone can reverse analgesia induced by acupuncture suggests that the release of endogenous opiates is one factor. ${ }^{18} 19$ Both the time delay before the onset of acupuncture analgesia and the prolonged effect afterwards indicate the release of some humoral factor. Further evidence is provided by a report of an increase in material with opioid activity in lumbar cerebrospinal fluid after electroacupuncture using surface electrodes; but again the material was not characterised, being measured by a relatively non-specific radioreceptor assay. ${ }^{20}$

The successful treatment of withdrawal symptoms in heroin addicts by electroacupuncture was first reported by 'Wen and Cheung in 1973. ${ }^{21}$ If indeed acupuncture analgesia operates through the release of endogenous opiates treatment of the withdrawal syndrome in addicts by electroacupuncture may be effective through a similar mechanism. In a recent study using a specific radioimmunoassay for methionine-enkephalin, basal concentrations were low in the cerebrospinal fluid of addicts showing withdrawal symptoms, but they rose during electroacupuncture treatment that abolished the symptoms. ${ }^{22}$ Blood concentrations did not alter. This was the first indication in vivo of the presence of methionine-enkephalin in human body fluids as measured by a specific assay system. Though long-term heroin abuse has been thought to be possibly associated with suppression of $\beta$-endorphin as well as methionine-enkephalin, these workers found that concentrations of $\beta$-endorphin were surprisingly high in both the blood 
and the cerebrospinal fluid and were not affected by acupuncture. Thus the withdrawal syndrome does not seem to be due to deficiency of $\beta$-endorphin; instead the receptor mechanisms may have been altered by the heroin abuse, producing a desensitisation to opiates. If this is so then the receptors for $\beta$-endorphin and methionine-enkephalin must be differently affected.

In this issue ( $\mathrm{p}$ 758) P T Pullan and his colleagues report the presence of methionine-enkephalin and $\beta$-endorphin (as well as $\beta$-lipotropin and ACTH) in carcinoid tumours in three patients with the ectopic ACTH syndrome. Higher concentrations of the brain opiate peptides were found in the draining blood than in the periphery, suggesting active secretion. Possibly these tumours were releasing behaviour-modifying products, including methionine-enkephalin and $\beta$-endorphin, which could not only suppress pain produced by the tumour and produce psychiatric manifestations but also modify the clinical presentation. Certainly the psychiatric symptoms resolved with removal of the tumour; and comparison of the pain thresholds of any further patients before and after operation will be interesting. Such tumours may also containand be producing-psychoactive substances that have not been detected but might be responsible for some of the varied clinical syndromes of malignancy, which can include psychiatric disturbances in the absence of cerebral metastases. We have become accustomed to the idea that neoplasia may produce ill health through the metabolic effects of tumour products secreted inappropriately, such as ACTH or vasopressin. This might be termed "biochemical malignancy." The finding that behaviour-modifying brain peptides may be secreted by non-neuronal tumours raises the possibility that mental changes in patients with cancer may not necessarily be due to metastases but may instead be due to the central effects of brain hormones secreted ectopically-a syndrome of "psychiatric malignancy."

${ }^{1}$ Hughes J, Smith TW, Kosterlitz HW, Fothergill LA, Morgan BA, Morris HR. Identification of two related pentapeptides from the brain with potent opiate agonist activity. Nature $1975 ; 258: 577-9$.

${ }^{2}$ Ling $\mathrm{N}$, Burgus $\mathrm{R}$, Guillemin $\mathrm{R}$. Isolation, primary structure, and synthesis of alpha-endorphin and gamma-endorphin, two peptides of hypothalamic-hypophysial origin with morphinomimetic activity. Proc Natl Acad Sci 1976;73:3942-6.

3 Frederickson RC. Enkephalin pentapeptides-a review of current evidence for a physiological role in vertebrate neurotransmission. Life $S c i$ $1977 ; 21: 23-42$.

${ }^{4}$ Belluzzi JD, Grant N, Garsky V, Sarantakis D, Wise CD, Stein L. Analgesia induced in vivo by central administration of enkephalin in rat. Nature 1976;260:625-6.

${ }^{5}$ Loh HH, Tseng LF, Wei E, et al. Beta-endorphin is a potent analgesic agent. Proc Natl Acad Sci 1976;73:2895-8.

6 Jacob JJ, Tremblay EC, Colombel M-C. Facilitation de réactions nociceptives par la naloxone chez la souris et chez le rat. Psychopharmacologia $1974 ; 37: 217-23$.

${ }^{7}$ Buchsbaum MS, Davis GC, Bunney WE. Naloxone alters pain perception and somatosensory evoked potentials in normal subjects. Nature 1977;270:620-2.

${ }^{8}$ Levine JD, Gordon NC, Jones RT, Fields HL. The narcotic antagonist naloxone enhances clinical pain. Nature 1978;272:826-7.

${ }^{9}$ Levine JD, Gordon NC, Fields HL. The mechanism of placebo analgesia. Lancet 1978 ;ii :654-7.

10 Guillemin R, Vargo T, Rossier J, et al. $\beta$-endorphin and adrenocorticotropin are secreted concomitantly by the pituitary gland. Science $1977 ; 197: 1367-9$

11 Clement-Jones V, Lowry PJ, Rees LH, Besser GM. Met-enkephalin circulates in human plasma. Nature 1980;283:295-7.

12 Reynolds DV. Surgery in the rat during electrical analgesia induced by focal brain stimulation. Science 1969;164:444-5.

13 Akil H, Mayer DJ, Liebeskind JC. Antagonism of stimulation-produced analgesia by naloxone, a narcotic antagonist. Science 1976;191:961-2.

${ }^{14}$ Hosobuchi Y, Adams JE, Linchitz R. Pain relief by electrical stimulation of the central gray matter in humans and its reversal by naloxone. Science 1977;197:183-6.

15 Jacquet YF, Lajtha A. Paradoxical effects after microinjection of morphine in the periaqueductal gray matter in the rat. Science $1974 ; \mathbf{1 8 5}: 1055-7$.
16 Akil H, Richardson DE, Hughes J, Barchas JD. Enkephalin-like material elevated in ventricular cerebrospinal fluid of pain patients after analgesic focal stimulation. Science $1978 ; 201: 463-5$.

17 Akil H, Richardson DE, Barchas JD, Li CH. Appearance of betaendorphin-like immunoreactivity in human ventricular cerebrospinal fluid upon analgesic electrical stimulation. Proc Natl Acad Sci 1978; 75:5170-2.

${ }^{18}$ Mayer DJ, Price DD, Rafii A. Antagonism of acupuncture analgesia in man by the narcotic antagonist naloxone. Brain Res 1977;121:368-72.

19 Pomeranz B, Chiu D. Naloxone blockade of acupuncture analgesia: endorphin implicated. Life $S_{c i}$ 1976;19:1757-62.

${ }^{20}$ Sjölund B, Terenius L, Eriksson M. Increased cerebrospinal fluid levels of endorphins after electro-acupuncture. Acta Physiol Scand 1977; 100:382-4.

21 Wen HL, Cheung SYC. Treatment of drug addiction by acupuncture and electrical stimulation. Asian f Med 1973;9:138-41.

22 Clement-Jones V, Mcloughlin L, Lowry PJ, Besser GM, Rees LH, Wen HL. Acupuncture in heroin addicts: changes in met-enkephalin and $\beta$-endorphin in blood and cerebrospinal fluid. Lancet 1979;ii:380-2.

\section{Haemoptysis}

Despite the decline in the prevalence of tuberculosis and bronchiectasis, haemoptysis remains a common diagnostic problem. A careful history may be helpful, including details of any recent dental treatment or general anaesthetics. In children another possibility is inhalation of sweets or foodstuffs. The history and clinical examination will help to exclude spurious haemoptysis from epistaxis, gingival bleeding, or haematemesis; and the first investigation should be a chest $x$-ray examination. Most patients with true haemoptysis have an underlying pulmonary infarct, pneumonia, or acute bronchitis, and usually a history of lung disease with associated radiographic abnormalities. Where an abnormality is visible on the $x$-ray film and these common causes seem unlikely it is logical to proceed straight to bronchoscopy. In patients who have a history of tuberculosis or bronchiectasis with recurrent haemoptysis this may sometimes be unnecessary, but these patients-like any others-can develop a bronchial carcinoma.

The problem group of patients has always been those with haemoptysis and apparently normal chest $x$-ray appearances who have no history of chest disease or recent acute illness. ${ }^{1}$ Hitherto, where initial cytological examination of the sputum has given negative results the most common practice has been to do further chest $x$-ray examinations after one month and at intervals for the next year or two. Longer follow-up has proved unrewarding. ${ }^{2}$ Few clinicians proceeded routinely to rigid bronchoscopy for these patients. The fibreoptic bronchoscope, however, has led to changes in clinical practice in these circumstances. ${ }^{3}$ Because it can be introduced under local anaesthesia with simple premedication and without hospital admission it has reduced the demands on services. The trachea and main bronchi can be inspected easily for the source of the haemoptysis and good specimens can be obtained for cytological examination.

A recent American study investigated the factors in patients with haemoptysis that indicated a high probability of malignant disease of the lung. ${ }^{4}$ An abnormality in the chest $x$-ray film and haemoptysis lasting longer than a week in patients over 40 years of age were associated with underlying neoplasms and were regarded as factors calling for bronchoscopy. In the absence of all these three factors a policy of observation without bronchoscopy was advocated. Because of the ease of fibreoptic bronchoscopy current practice in Britain is probably different. Neither a tracheal carcinoma nor a bronchial adenoma is usually visible in the chest radiograph. The added 